outcome, occlusion rate and reoperation. Moreover, clipping and stent-assisted coiling all showed higher occlusion rates compared with coiling alone.

Conclusion Definitely, EVT is the mainstream treatment for paraclinoid aneurysms. Surgical clipping showed a higher complication risk than EVT. Clipping might be considered to be an alternative option for paraclinoid aneurysms if EVT are for hard hurdle because many complications were temporary, and occlusion tendency of clipping was also good.

Disclosures S. Chung: None. S. Chang: None.

\section{E-210 AN IN VITRO STUDY OF INTRANEURYSMAL PRESSURE INCREASES DURING CONTRAST INJECTIONS IN CEREBRAL ANGIOGRAPHY}

${ }^{1} S$ Marfoglio, ${ }^{1}$ B Kovarovic, ${ }^{2}$ W Hou, ${ }^{3} \mathrm{D}$ Fiorella, ${ }^{3} \mathrm{C}$ Sadasivan*. ${ }^{*}$ Biomedical Engineering, Stony Brook University, Stony Brook, NY; ${ }^{2}$ Family, Population and Preventive Medicine, Stony Brook University, Stony Brook, NY; ${ }^{3}$ Neurological Surgery, Stony Brook University, Stony Brook, NY

\subsection{6/neurintsurg-2020-SNIS.241}

Introduction During angiography, the contrast bolus injected into a vessel can cause substantial changes in baseline pressures and flows. ${ }^{1}$ There have been sparse case reports about aneurysmal re-bleeds during angiography. ${ }^{2}$ A physician survey of injection rates used during angiography showed that $81 \%$ of respondents considered the impact of injection rate on aneurysm rupture to be negligible. ${ }^{3}$ The goals of this study were to record intraneurysmal pressure changes during contrast injections and to evaluate the effect of injection conditions on intraneurysmal pressure changes.

Methods A silicone replica of a complete circle of Willis model with ophthalmic, anterior communicating, and basilar tip aneurysms was connected to a pulsatile flow pump (Vascular Simulations, Stony Brook, NY, figure 1). Catheters were placed in either the right internal carotid or right vertebral artery and a total of 144 injections were performed by varying 4 different parameters: Catheter Size, Injection Rate, Injection Time, mean Baseline Blood Flow rate (table). The effect of each of the four injection parameters on percentage increase in aneurysm pressures was statistically assessed using ANCOVA; baseline pressure was considered a covariate.

Results The mean intraneurysmal pressure during injection $(84.5 \pm 10.8 \mathrm{mmHg})$ was significantly higher than the mean pressure before injection $(80.4 \pm 10.6 \mathrm{mmHg})(\mathrm{p}<0.0001)$. The percentage increase in mean and systolic aneurysm pressures was $5.1 \pm 3.6 \%$ and $3.4 \pm 2.6 \%$, respectively. The percentage increase in aneurysm pressure was significantly greater $(p<0.0001)$ at high Injection Rates versus low Injection Rates $(6.6 \pm 4 \%$ versus $3.7 \pm 3 \%)$. Increase in aneurysmal pressure was also significantly affected by Catheter Size in most of the statistical comparisons $(p<0.0001)$. Injection Time $(p>0.2)$ and Baseline Blood Flow rate $(p>0.3)$ had no significant effect on the increase in intraneurysmal pressure.
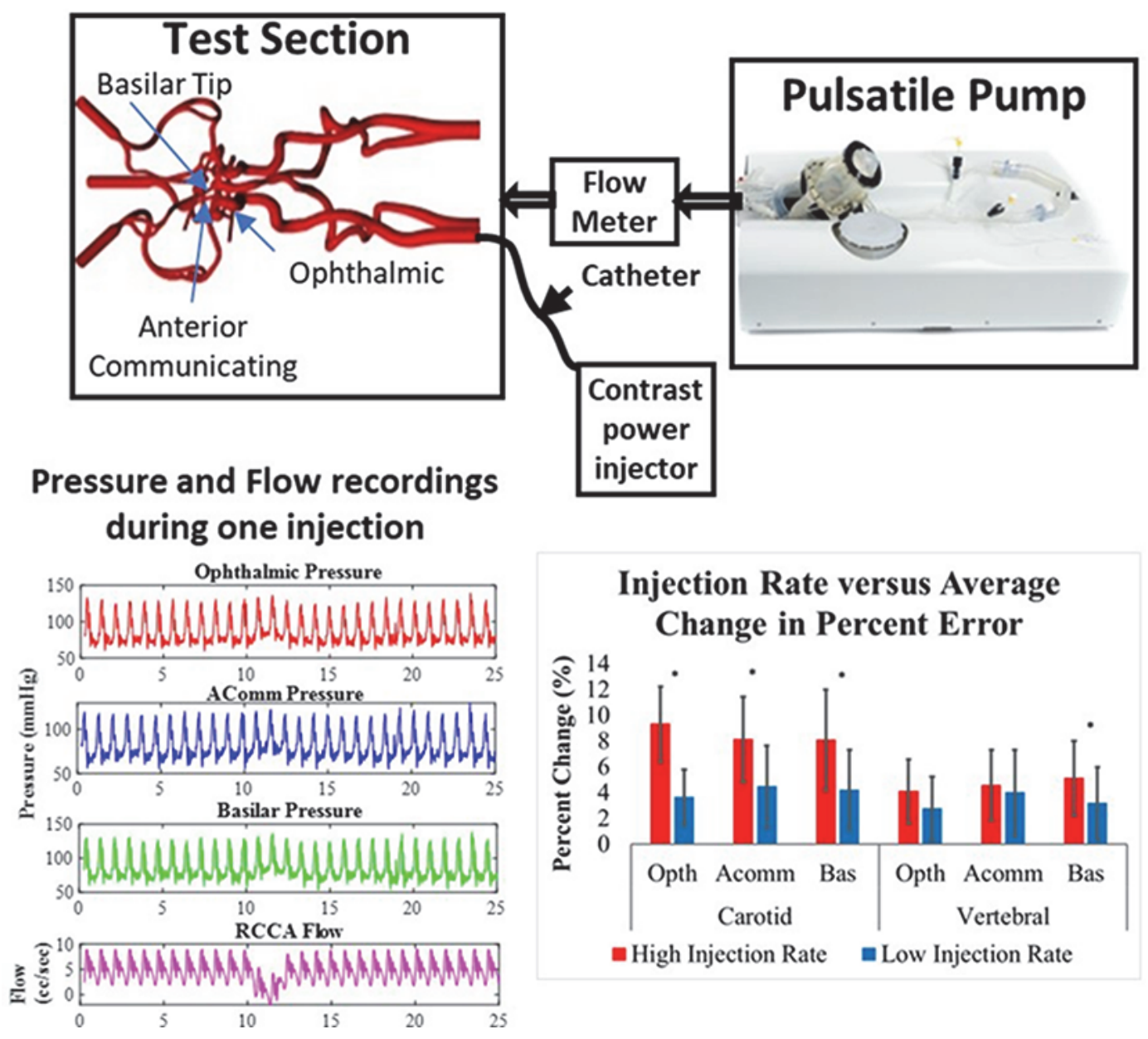

Injection Rate versus Average Change in Percent Error

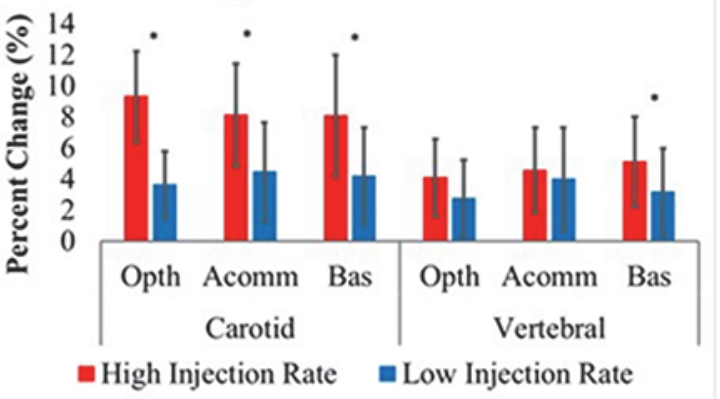

Abstract E-210 Figure 1 
Conclusion Our results suggest that higher contrast Injection Rates can significantly increase intraneurysmal pressures during angiography. Whether a $\sim 5 \%$ increase in intraneurysmal pressure can cause aneurysm rupture needs to be evaluated. Angiography studies need to be conducted in vivo to verify these findings.

\section{REFERENCES}

1. Kovarovic B, et al. Cardiovasc Eng Technol 2018;9(2):226-239.

2. Celtikci $P$, et al. Turk Neurosurg 2018:28(6):1009-1012.

3. Yousem, DM, et al. AJNR 2001;22:1838-1840.

Disclosures S. Marfoglio: 5; C; Vascular Simulations, Inc. B. Kovarovic: None. W. Hou: None. D. Fiorella: 4; C; Vascular Simulations Inc. C. Sadasivan: 2; C; Vascular Simulations Inc. 4; C; Vascular Simulations Inc. 6; C; Vascular Simulations Inc.

\section{E-211 ANEURYSM SIZE AND IMPACT ON MIDDLE CEREBRAL ARTERY PRESSURE: CHANGES FOLLOWING FLOW DIVERSION}

${ }^{1} \mathrm{D}$ Brunozzi*, ${ }^{2} \mathrm{~A}$ See, ${ }^{2} \mathrm{~A}$ Alaraj. ${ }^{1}$ Neurosurgery, university of illinois at chicago, Chicago, IL; ${ }^{2}$ Neurosurgery, university of illinois at chicago, Chicago, IL

10.1136/neurintsurg-2020-SNIS.242

Background The impact of cerebral aneurysm size on distal intracranial hemodynamics such as pressure is not completely understood, either before or after flow diversion (FD).

Objective To assess the impact of aneurysm size on distal pressure before and after FD.METHODS Beginning in December 2015, prospective measurement of middle cerebral artery (MCA) pressure was completed in consecutive patients with unruptured cerebral aneurysms in the ophthalmic to communicating segments of the internal carotid artery which were treated with a single FD. Pressure was recorded at the M1 segment ipsilateral to the cerebral aneurysm. Ratio of MCA to radial arterial pressure (pressure ratio, PR) controlled for variations in systemic blood pressure. Correlation between aneurysm size and MCA PR was assessed before and after treatment.

Results 29 aneurysms were treated in 27 patients. Mean aneurysm size was $7.4 \mathrm{~mm}(2-27 \mathrm{~mm})$. Aneurysm size correlated linearly with systolic PR $(1 \%$ per $\mathrm{mm}, \mathrm{p}=0.001$, $\mathrm{r}^{2}=0.33$; figure $\left.1 \mathrm{~A}\right)$ and mean $\mathrm{PR}(0.6 \%$ per $\mathrm{mm}, \mathrm{p}=0.03$, $\mathrm{r}^{2}=0.17$; figure $\left.1 \mathrm{~B}\right)$. After $\mathrm{FD}$, aneurysm size continued to have a linear correlation with the systolic PR $(1 \%$ per $\mathrm{mm}$, $\mathrm{p}=0.004, \mathrm{r}^{2}=0.28$; figure $\left.1 \mathrm{~A}\right)$, but not with the mean PR $\left(0.4 \%\right.$ per $\mathrm{mm}, \mathrm{p}=0.15, \mathrm{r}^{2}<0.1$; figure $\left.1 \mathrm{~B}\right)$.

Conclusion Aneurysm size affects distal hemodynamics: patients with larger aneurysms have increased systolic and mean MCA PR. After FD, mean MCA PR no longer associates with the aneurysm size, suggesting an effect of the FD on distal intracranial hemodynamics.

Disclosures D. Brunozzi: None. A. See: None. A. Alaraj: 1; C; NIH. 2; C; Cerenovus, Siemens.

\section{E-212 PULSERIDER IN THE TREATMENT OF WIDE-NECK BIFURCATION ANEURYSMS: INTERIM RESULTS OF THE NAPA TRIAL}

${ }^{1} \mathrm{~A}$ Spiotta* ${ }^{*},{ }^{2} \mathrm{~K}$ Ebersole, ${ }^{3} \mathrm{~J}$ Lena, ${ }^{4} \mathrm{R}$ Starke, ${ }^{5} \mathrm{R}$ De Leacy, ${ }^{6} \mathrm{~A}$ Puri, ${ }^{7} \mathrm{D}$ Yavagal, ${ }^{8} \mathrm{~B}$ Bohnstedt, ${ }^{9} \mathrm{~L}$ Rangel-Castilla, ${ }^{10} \mathrm{~A}$ Cheema, ${ }^{11} \mathrm{~J}$ Davies, ${ }^{12} \mathrm{~K}$ de Macedo Rodrigues, ${ }^{13} \mathrm{~J}$ Grossberg, ${ }^{14} \mathrm{~B}$ Howard, ${ }^{15} \mathrm{C}$ Kellner, ${ }^{16} \mathrm{G}$ Lanzino, ${ }^{17} \mathrm{~S}$ Tateshima. ${ }^{1}$ Department of Neurosurgery, Medical University of South Carolina, Charleston, SC; ${ }^{2}$ Department of Vascular and Endovascular Neurosurgery, University of Kansas Medical Center, Kansas City, KS; ${ }^{3}$ Department of Neruosurgery, Medical University of South Carolina, Charleston, SC; ${ }^{4}$ University of Miami - Jackson Memorial Hospital, Miami, FL; ${ }^{5}$ Mount Sinai Hospital, New York, NY; ${ }^{6}$ New England Center for Stroke Research, Department of Radiology, University of Massachusetts Medical Center, Worcester, MA; ${ }^{7}$ Department of Neurosurgery, University of Miami - Jackson Memorial Hospital, Miami, FL; ${ }^{8}$ Department of Neurological Surgery, Indiana University School of Medicine, Indianapolis, IN; ${ }^{9}$ Mayo Clinic, Rochester, MN; ${ }^{10}$ University of Oklahoma Health Sciences Center, Oklahoma City, OK; ${ }^{11}$ Department of Neurosurgery, University of Buffalo Medical Center, Buffalo, NY; ${ }^{12}$ University of Massachusetts Medical Center, Worcester, MA; ${ }^{13}$ Neurosurgery and Radiology, Emory Clinic, Atlanta, GA; ${ }^{14}$ Department of Neurosurgery, Emory Clinic - School of Medicine Faculty, Atlanta, GA; ${ }^{15}$ Department of Neurosurgery, Mount Sinai Hospital, New York, NY; ${ }^{16}$ Departments of Surgery, Radiology, and Neurosurgery, Mayo Clinic, Rochester, MN; ${ }^{17}$ Department of Radiological Sciences, University of California, Los Angeles, Diagnostic and Interventional Radiology Diagnostic Radiology, Los Angeles, CA

\subsection{6/neurintsurg-2020-SNIS.243}

Introduction The NAPA study was a prospective, multicenter, single-arm IDE trial of the PulseRider device as an adjunctive treatment in conjunction with coil embolization of unruptured wide-neck bifurcation aneurysms which was electively discontinued for reasons not related to the safety or performance of the device. We report on the available enrollments in NAPA and their available follow up.

Methods The PulseRider device was implanted in 18/21 enrollments. Available core-lab adjudicated data from the first 18 implants is presented. Of those, 5 have one year follow up data available. Of the 3 device implantation failures, the locations included basilar (1), carotid terminus (1), and ACOMM (1).
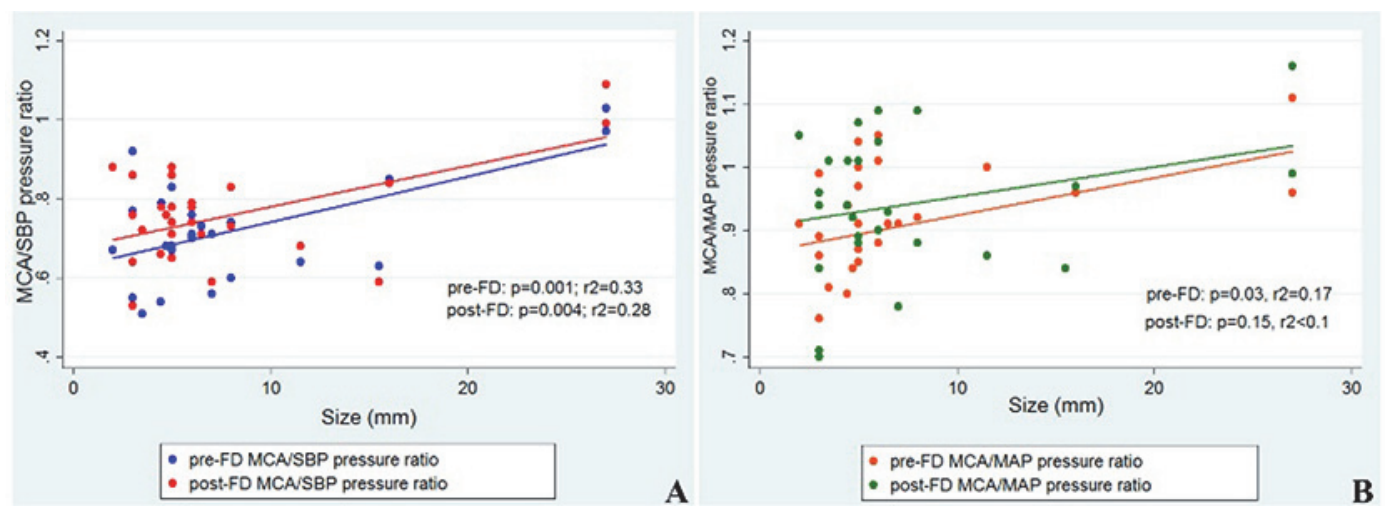

\section{Abstract E-211 Figure 1}

